\title{
OFICINAS DE CUIDADO: UM RELATO DE EXPERIÊNCIA COM OS TRABALHADORES DA SAÚDE DA FAMÍLIA
}

\section{Health care workshops: an experience report with family health workers \\ Talleres de cuidado: relato de experiencia de trabajadores de la salud de la familia}

\author{
Luana Fagundes Galiski iD \\ Universidade do Vale do Itajaí - UNIVALI - Itajaí (SC) - Brasil
}

\author{
Carlos Alberto Severo Garcia Júnior (iD \\ Universidade Federal de Santa Catarina - UFSC - Araranguá (SC) - Brasil
}

Letícia Blasius da Cunha (D)

Universidade do Vale do Itajaí - UNIVALI - Itajaí (SC) - Brasil

Thais Bolognini iD

Universidade do Vale do Itajaí - UNIVALI - Itajaí (SC) - Brasil

Vinicius Campos iD

Universidade do Vale do Itajaí - UNIVALI - Itajaí (SC) - Brasil

\section{RESUMO}

Objetivo: Relatar o processo de construção e realização de oficinas de cuidado em saúde com trabalhadores da Estratégia de Saúde da Família. Síntese dos dados: Relato de experiência realizada com trabalhadores de Unidades Básicas de Saúde de um município da região do Vale do Itajaí, Santa Catarina, Brasil. Utilizaram-se, para a promoção da reflexão e de um olhar ampliado no cuidado em saúde, no primeiro semestre de 2018, oficinas mediadas por residentes multiprofissionais em Atenção Básica/ Saúde da Família da Universidade do Vale do Itajaí. Conclusão: Promoveu-se um espaço de reflexão e mobilização para o cuidado em saúde, utilizando a escuta como reestruturação produtiva em saúde, por meio da atividade realizada, sendo possível compreender o quanto a residência pode contribuir para o fortalecimento do serviço em saúde, orientando sobre o cuidado em saúde desses trabalhadores. Assim, é necessário que essas estratégias metodológicas sejam cada vez mais acatadas pelos coordenadores das residências e pelos gestores.

Descritores: Educação em Saúde; Atenção Primária à Saúde; Saúde do Trabalhador.

\section{ABSTRACT}

Objective: To report the processes of development and implementation of health care workshops with Family Health Strategy professionals. Data synthesis: This report experience study was conducted with professionals of Primary Health Care Centers of a municipality in the Vale do Itajai region, Santa Catarina, Brazil. Reflection on and a broader view of health care was promoted by workshops held in the first half of 2018 mediated by multi-professional Primary Care/Family Health residents of the University of Vale do Itajaí. Conclusion: The workshops made room for reflection on and mobilization for health care by using listening as a productive tool for restructuring health. It was possible to understand how much residency can contribute to strengthening the health care service by providing guidelines on health care for health workers. Thus, such methodological strategies should be increasingly embraced by residency coordinators and managers.

Descriptors: Health education; Primary Health Care; Occupational Health.

\section{RESUMEN}

Objetivo: Relatar el proceso de construcción y realización de talleres de cuidado para la salud de trabajadores de la Estrategia Salud de la Familia. Síntesis de los datos: Relato de experiencia de trabajadores de las Unidades Básicas de Salud de un municipio de la región del Vale de Itajaí, Santa Catarina, Brasil. Para la promoción de la reflexión y de una mirada ampliada

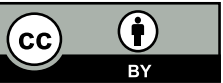


del cuidado en salud se utilizaron en el primer semestre de 2018 de talleres mediados por residentes multiprofesionales de la Atención Básica/Salud de la Familia de la Universidad del Vale de Itajaí. Conclusión: Se ha promovido un espacio de reflexión y movilización sobre el cuidado en salud utilizándose la escucha para la reestructuración productiva de la salud a través de la actividad realizada lo que ha permitido la comprensión de lo mucho que la residencia puede contribuir para el fortalecimiento del servicio de salud orientando sobre el cuidado de la salud de trabajadores sanitarios. Así, es necesario que las estrategias metodológicas sean cada vez más aceptadas por los coordinadores de las residencias y por los gestores.

Descriptores: Educación en Salud; Atención Primaria de Salud; Salud Laboral.

\section{INTRODUÇÃO}

O modo de trabalho absorve e exige, a cada dia, energia física e psicológica de seus trabalhadores, pois está baseado na produção, que pode levar o trabalhador, rapidamente, ao desgaste e à exaustão, somando-se a isso a exposição diária de fatores de risco a sua saúde. Por outro lado, a falta de trabalho é também um fator de adoecimento $^{(1)}$.

O homem está em um contínuo processo de autoconstrução, possibilitado por sua atividade essencial, o trabalho ${ }^{(2)}$. É por meio dessa atividade vital que o homem objetiva o seu espírito no mundo e materializa em objetos suas inquietações, ideias e sentimentos, tendo então, como fruto, os bens materiais necessários à existência, assim como toda a riqueza social( ${ }^{(3)}$.

A Política Nacional de Promoção da Saúde (PNPS) descreve o trabalho como um dos determinantes do processo saúde-adoecimento, incluindo em seu objetivo geral que as condições de trabalho estão diretamente relacionadas à qualidade de vida, aos riscos à saúde e a vulnerabilidades ${ }^{(4)}$. Sendo assim, a Política Nacional de Saúde do Trabalhador (PNST) tem como enfoque a promoção e a proteção da saúde dos trabalhadores e a redução da morbimortalidade decorrente dos modelos de desenvolvimento e dos processos produtivos, com ações de intervenção sobre os fatores determinantes da saúde dos trabalhadores ${ }^{(5)}$.

O trabalho em saúde é reflexo do modelo organizacional dos serviços de saúde, que, por sua vez, são caracterizados por estruturas hierarquizadas e verticalizadas, sendo estruturados nas relações humanas instituídas por seus diversos atores sociais e estando sujeitos a sucessos e percalços, especialmente pela realização de tarefas com alto grau de exigência e responsabilidade ${ }^{(6)}$.

Dentro do cenário da Atenção Primária à Saúde (APS), diversos são os fatores que podem interferir na saúde dos trabalhadores da área de saúde, especificamente na Equipe de Saúde da Família (ESF), participantes ativos desse complexo contexto de saúde ${ }^{(7)}$. Alguns exemplos, e que tendem a piorar com o passar dos anos, são: a falta de recursos humanos e materiais, a estrutura física, as diversas formas de inserção no mercado de trabalho (marcadas pela fragilização de políticas públicas e organizacionais direcionadas a este setor) e, até mesmo, os problemas sociais da população. Esses fatores podem levar os profissionais a vivenciarem o sofrimento, a dor e o adoecimento físico e psíquico, sendo nesse conflituoso cenário que a degradação da saúde pode ocorrer ${ }^{(6)}$.

Apresentam-se, dessa forma, elevadas incidências de problemas de saúde, como depressão, ansiedade, amnésia, dificuldade de concentração, queixas somáticas, insônia, fadiga e irritabilidade ${ }^{(8)}$. Além disso, o trabalho na ESF foi relatado como o principal causador de sofrimento psíquico, sobretudo por problemas relacionados à infraestrutura, como ambientes abafados e espaços inapropriados para atendimento. Também os relacionados à organização do trabalho, como a falta de funcionários e a consequente sobrecarga de trabalho, com a realização de múltiplas tarefas, e dificuldade de relacionamento entre os profissionais da equipe ${ }^{(9)}$.

Assim, os trabalhadores de saúde estão constantemente expostos e susceptíveis a riscos ocupacionais durante o desempenho de suas atividades, qualquer que seja sua categoria profissional, advindos, principalmente, pela carência de recursos para a realização do trabalho, ao desgaste emocional e à violência física e moral, frequentemente observados nesse contexto laboral ${ }^{(10)}$.

Estando o trabalho relacionado ao adoecimento e vice-versa, fazem-se necessárias, no Sistema Único de Saúde (SUS), práticas que promovam a integralidade do cuidado aos profissionais de saúde. Frente ao exposto, a PNPS define, como um de seus objetivos, que sejam promovidos espaços de educação em saúde e formação profissional, além de capacitações específicas em promoção da saúde para trabalhadores, gestores e cidadãos ${ }^{(11)}$.

Nessa mesma linha de pensamento da PNPS, no intuito de construir uma política de valorização do trabalhador do Sistema Único de Saúde (SUS), o Ministério da Saúde aponta a Educação Permanente em Saúde (EPS) como 
estratégia fundamental para a recomposição das práticas de formação, bem como das práticas pedagógicas e de saúde ${ }^{(12)}$.

A EPS tem como foco promover o ensino e a aprendizagem no cotidiano de serviço dos profissionais da saúde, deve se basear na dialogicidade e problematização da realidade e na construção conjunta e criativa de soluções pensadas por sujeitos que se desenvolvem e se emancipam durante o processo, visando à transformação das práticas de saúde de acordo com os princípios e diretrizes do SUS ${ }^{(13)}$.

O modelo do profissional de saúde atual, promovido pelo mundo do trabalho, envolve as concepções de saúde e educação e necessita ser experienciada no processo de formação profissional, expandindo as probabilidades de compartilhamento de saberes e práticas. Sendo assim, o estudo tem sua relevância, pois pretende possibilitar uma formação de qualidade aos profissionais da Residência Multiprofissional em Saúde (RMS), para, dessa forma, contribuir e fortalecer a mudança do desenho tecnoassistencial do SUS. Desde 2002, o Ministério da Saúde vem apoiando e financiando tais residências ${ }^{(14)}$, a qual, por serem uma formação em saúde em nível de pós-graduação, tem como principal característica promover, através do trabalho em saúde, o ensino em serviço ${ }^{(15)}$.

A Portaria n 1.248, de 24 de junho de 2013, cria a Estratégia de Qualificação das Redes de Atenção à Saúde (RAS) por meio do incentivo à formação de especialistas na modalidade de residência em áreas estratégicas do Sistema Único de Saúde, e tem como um de seus objetivos o aprimoramento técnico-científico necessário para a qualificação da atuação dos profissionais ${ }^{(16)}$.

As RMS é um espaço privilegiado de formação e gestão de pessoas para atuação no SUS, sendo baseada em uma formação multiprofissional e interdisciplinar, ou seja, uma formação que compartilha saberes e que possibilita a socialização de conhecimentos e de linguagens no ato da formação e do próprio trabalho em saúde ${ }^{(15)}$.

Nesse sentido, o relato a ser descrito provém de ações executadas na atuação de alunos da Residência Multiprofissional em Saúde da Família/Atenção Básica. A realização desse estudo deu-se pela possibilidade de proporcionar um momento de reflexão para os profissionais sobre o seu cuidado em saúde, discutir probabilidades de cuidado e estimular o desenvolvimento dessas práticas no cotidiano. Um grande facilitador dessa experiência está no fato da Residência Multiprofissional em Saúde estar inserida dentro do campo de prática dessas equipes, havendo um processo prévio de formação de vínculo e confiança.

Com base no exposto, o presente artigo objetiva relatar o processo de construção e realização de oficinas de cuidado em saúde com trabalhadores da Estratégia de Saúde da Família.

\section{SÍNTESE DOS DADOS}

Trata-se de um relato de experiência resultante do trabalho de alunos de Residência Multiprofissional em Saúde (RMS), vinculados à formação e às práticas de produção de saúde no SUS, materializado a partir da construção e realização de oficinas de educação permanente em saúde, efetivadas em Unidades Básicas de Saúde (UBS) de um município da região do Vale do Itajaí, Santa Catarina, Brasil.

A Unidade Básica de Saúde (UBS) está inserida no SUS como base da Atenção Primária, sendo o contato preferencial dos usuários, a principal porta de entrada e o centro de comunicação com toda a Rede de Atenção à Saúde (RAS). De modo a facilitar seu acesso, está instalada perto de onde as pessoas moram, trabalham, estudam, vivem e, com isso, desempenha um papel central na garantia de acesso à população a uma atenção à saúde de qualidade $^{(17)}$.

A atividade de cuidado em saúde foi planejada e desenvolvida pelo programa de Residência Multiprofissional em Atenção Básica/Saúde da Família, da Universidade do Vale do Itajaí, por alunos de Fisioterapia, Psicologia, Nutrição e Educação Física, com apoio de tutor/professor da residência. As UBS são os locais de prática dos residentes e, antes da realização das atividades, estes realizaram o convite aos trabalhadores presentes em cada unidade da ESF. Todos se dispuseram a participar após conhecimento dos objetivos das atividades. Os trabalhadores de saúde que participaram foram médicos, enfermeiros, técnicos de enfermagem, cirurgiões-dentistas e agentes comunitários de saúde.

As oficinas fizeram parte de um conjunto de ações de educação permanente em saúde e apoio institucional relacionadas ao II Seminário de Saúde do Trabalhador (II SST), no município de Itapema, na região do Vale do Itajaí, a partir das necessidades relatadas durante o evento. Esse seminário, ocorrido em abril de 2018, teve por objetivo refletir sobre os aspectos que influenciavam negativamente na saúde dos trabalhadores. O II SST foi considerado um instrumento disparador de mudanças, desde o princípio, pois, abriu espaço para ações de educação permanente para os trabalhadores da saúde. 
As oficinas de educação permanente em saúde, intituladas de "caminhos do cuidado", foram planejadas durante o mês de maio e executadas em junho de 2018, nas Unidades Básicas de Saúde (UBS) do município em questão, contando com o apoio da gestão e do Programa de Saúde do Trabalhador (PST). Ao total, realizaram-se dez oficinas com os trabalhadores de saúde de dez UBS. Cada encontro teve duração de, aproximadamente, uma hora e meia, abrangendo todas as UBS, que possuem um total de 14 Estratégias Saúde da Família (ESF).

As oficinas são espaços amplamente utilizados nas áreas da saúde e educação, pois possibilitam, aos que delas participam, um lugar de fala, expressão e acolhimento, sendo práticas sociais que proporcionam espaços de construção acerca dos temas discutidos ${ }^{(18)}$.

A cada encontro, antes do início das oficinas, realizava-se uma contextualização, explicando o objetivo de sua realização e a relação com o II SST já descrito, e o produto final desse evento foi a construção dos "itinerários terapêuticos" dos trabalhadores da saúde. Itinerários terapêuticos é um termo utilizado para designar as atividades desenvolvidas pelos indivíduos em busca de tratamento para a doença ou aflição. Portanto, se a pretensão é saber de que modo o trabalhador está adoecendo ao exercer suas atividades laborais, é preciso que sejam escutados e possam relatar como estão vivenciando o trabalho, quais são as estratégias de enfrentamento utilizadas para responder às demandas do trabalho e, também, como os equipamentos de saúde estão ou não dando condições para que possam realizar o que lhes é demandado(19).

Sendo assim, (re)conhecer, por meio de um itinerário terapêutico traçado pelos profissionais de saúde, o seu processo relacional com o trabalho, parte do pressuposto de que, para compreender a relação entre trabalho e saúde, é necessário basear-se na vivência do sujeito. Desse modo, o itinerário terapêutico é um dispositivo revelador da cultura do cuidado e do cuidado na e da cultura, retratando a multiplicidade de sua constituição, a qualidade das relações sociais e os potenciais de cuidado. Além disso, tenciona as práticas/noções construídas no campo da saúde sobre experiências de adoecimento, redes de apoio, acesso, adesão e escolhas terapêuticas, possibilitando o potencial analítico que tem em olhar para o processo de saúde-doença-cuidado ${ }^{(19)}$.

Relatar as práticas de cuidado e de gestão em saúde implica compreender a variedade de fatores que as constituem, como a complexidade das relações entre os trabalhadores, gestores e usuários dos serviços de saúde. Elaborar um processo de formação para trabalhadores de saúde requer estar atento a essa complexidade e fazer escolhas teórico-metodológicas que expressem um campo de interlocução entre os saberes. São necessários modos de fazer a formação que se construam a partir da integralidade e da indissociabilidade entre cuidar, gerir e formar, potencializando movimentos de mudança por meio da indagação dos modos instituídos ${ }^{(20)}$.

Dessa forma, a educação permanente em saúde estimula o empoderamento e a integração dos trabalhadores no ambiente de trabalho através da percepção das fragilidades no cuidado, estimulando a união entre os trabalhadores através de uma troca de saberes não hierarquizada. Essa modalidade de formação no próprio trabalhotem sido cada vez mais reconhecida ${ }^{(21)}$.

As dez oficinas realizadas foram efetivadas, cada uma, em uma UBS diferente e divididas em duas etapas: a primeira consistiu na realização da dinâmica do "baralho das emoções" para promover interação e descontração entre os participantes, na qual, cada indivíduo escolhia uma carta do baralho para definir suas emoções naquele momento e, posteriormente, compartilhar com o colega ao lado. Realizou-se a segunda etapa a partir de um "jogo de tabuleiro" contendo seis estações: mente, corpo, alimentação, relações, lazer e autocuidado. Essas estações eram escolhidas a partir das necessidades mais indicadas pelos trabalhadores na construção do itinerário terapêutico. A cada oficina alcançada, havia a participação de quatro residentes (fisioterapeuta, nutricionista, profissional de educação física e psicóloga) que atuavam, concomitantemente, como mediadores do processo de realização das oficinas, de acordo com sua área de conhecimento e as contribuições a serem agregadas em cada atividade.

A proposta lúdica das oficinas permitiu que seu desenvolvimento ocorresse de forma natural, facilitando a abordagem para os mediadores. A experiência mostrou que o momento da oficina foi válido para todos os envolvidos, promovendo um momento de discussão, reflexão e relaxamento sobre os diversos assuntos que surgiram nas oficinas. Ao final de cada encontro, realizava-se uma avaliação e entrega de uma lembrança para cada participante: sementes de girassol para plantio, simbolizando o início do crescimento de tudo que foi abordado durante as oficinas, e seu florescimento posterior.

Simbolicamente, acredita-se que o maior crescimento, durante todo esse processo, foi o da própria equipe dos residentes que planejou as oficinas, pois enquanto cada detalhe das oficinas era preparado, puderam refletir sobre o real significado do trabalho integrado em equipe, como o fato de serem profissionais de áreas distintas, mas se complementarem e encontrarem um denominador comum em prol dos trabalhadores da saúde. 
Sobre esse assunto ${ }^{(22)}$, a RMS foi percebida como uma importante contribuição para uma mudança de paradigma da atenção à saúde na ESF, visando à reorganização da atenção básica, pois o convívio diário dos trabalhadores com os residentes e suas práticas proporcionou constantes discussões entre eles e o trabalhador. Por outro lado, em outra pesquisa ${ }^{(23)}$, compreendeu-se que a integração entre residentes e profissionais do serviço ocorreu de forma insuficiente. Assim, um trabalho dessa natureza, como o das oficinas em questão, dá visibilidade ao trabalho dos residentes, nas UBS, junto a ESF.

A RMS proporciona adentrar espaços para conhecimento do funcionamento e de fluxos de diversos setores da saúde, entre eles, o PST, no qual iniciou-se o processo de desenvolvimento de uma ação para modificação do cenário dos trabalhadores. Como já citado, as oficinas surgiram após a realização do II Seminário de Saúde do Trabalhador, em abril de 2018. Entretanto este não foi o único disparador para essa experiência, visto que a imersão dos residentes no município proporcionou conhecimento prévio sobre o tema.

O aprender em serviço, ou a formação em serviço, é uma forma de construir conhecimentos e práticas na qual o desafio é articular a teoria com a prática cotidiana, significa viver em trânsito por entre esses dois campos. A residência propõe a experimentação de algo que parece difícil de alcançar: a tradução das teorias em práticas do cotidiano, o residente habita um lugar híbrido entre o ser trabalhador e o aprendiz, ao mesmo tempo em que traz um campo de conhecimento e de experimentações, que leva ao contexto de prática, oportunizando, aos trabalhadores com os quais está envolvido, novas experiências ${ }^{(24)}$.

A educação permanente em saúde tem como finalidade preparar e melhorar as habilidades dos profissionais de saúde frente ao desenvolvimento científico, tecnológico e organizacional, a fim de que sejam capazes de suprir as demandas e necessidades, tanto sociais como do próprio sistema de saúde ${ }^{(25)}$. Apresenta-se como uma importante estratégia de aperfeiçoamento do processo de trabalho e ferramenta de geração de mudanças proativas na gestão em saúde. Sendo assim, permite, na sua prática, transcender a unicidade e repetição das atividades educativas focalizadas, tecnicistas e desvinculadas das reais necessidades do cotidiano de trabalho, estabelecendo uma práxis inovadora e transformadora em saúde(26).

A relevância de ações como esta relatada consiste na necessidade, cada vez maior, de proporcionar espaços com perspectivas e olhares em relação a um mesmo ambiente de trabalho. Contudo acredita-se que experiências assim podem ser replicadas em diversos contextos na área da saúde e em demais áreas de atuação, pois a demanda por cuidado em saúde é crescente no mundo atual, basta um olhar atento e sensível aos sinais e sintomas que trabalhadores apresentam, que podem ser alterações na sua saúde.

Os resultados deste estudo conduzem à compreensão da realidade de ensino e assistência vivenciada pelos residentes, o que contribui agregando conhecimento no processo de formação profissional dos residentes para o SUS. O impacto dessa experiência pode se dar a partir da observação das Diretrizes da Política Nacional de Promoção da Saúde do Trabalhador do SUS, com vistas a elaboração dos planos, programas, projetos e ações de saúde voltados à população trabalhadora do SUS ${ }^{(27)}$.

Foi possível compreender o quanto a residência pode contribuir para o fortalecimento do serviço em saúde, orientando sobre o cuidado em saúde dos trabalhadores de saúde. Assim, é necessário que essas estratégias metodológicas sejam cada vez mais acatadas pelos coordenadores das residências e pelos gestores.

\section{CONCLUSÃO}

O presente relato descreveu uma experiência de construção e realização de oficinas de educação permanente em saúde, focadas no cuidado em saúde, com trabalhadores da Estratégia de Saúde da Família, vinculada à residência e construída através de diferentes olhares e de forma multiprofissional.

A prática contribuiu para o processo de valorização do profissional residente no município, mostrando aos demais profissionais e à gestão o quanto a residência pode contribuir para o fortalecimento do serviço em saúde e do PST do município. O apoio da gestão nesse processo ocorreu como forma de facilitação e articulação para o acesso das equipes de saúde, sendo esse processo de aproximação com os gestores uma oportunidade de crescimento profissional pela vivência dentro desses setores, tomando conhecimento de fluxos e recursos para a realização de ações no campo da saúde.

\section{CONFLITOS DE INTERESSE}

Os autores declaram a inexistência de conflitos de interesses durante a realização do estudo. 


\section{CONTRIBUIÇÕES}

Luana Fagundes Galiski contribuiu com elaboração e delineamento do estudo, análise e interpretação dos dados. Carlos Alberto Severo Garcia Júnior contribuiu com a aquisição, análise e interpretação de dados e a revisão do manuscrito. Letícia Blasius da Cunha, Thaís Bolognini e Vinicius Campos contribuíram com a revisão do manuscrito.

\section{FONTES DE FINANCIAMENTO}

Universidade do Vale do Itajaí, Programa de Saúde do Trabalhador do município de Itapema e Ministério da Saúde.

\section{REFERÊNCIAS}

1. Sampaio RSG, Carvalho RC, Rocha GS. Distúrbios psíquicos menores em trabalhadores que atuam na Estratégia de Saúde da Família: revisão integrativa. Anais do 210 Seminário de Iniciação Científica; 2018 Out 23-29; Feira de Santana, BA. [Internet]. [acesso em 2019 Abr 17]. Disponível em: http://periodicos.uefs.br/ index.php/semic/article/view/2558

2. Marx K. Manuscritos econômico-filosóficos. Ranieri J, tradutor. São Paulo: Boitempo; 2004.

3. Marx K, Engels F. A ideologia alemã. Enderle R, Schneider N, Martorano LC, tradutores. São Paulo: Boitempo; 2007.

4. Ministério da Saúde (BR). Secretaria de Vigilância em Saúde, Secretaria de Atenção à Saúde. Política Nacional de Promoção da Saúde. $3^{a}$ ed. Brasília: Ministério da Saúde; 2010.

5. Brasil. Ministério da Saúde. Portaria $n^{\circ} 1.823$, de 23 de agosto de 2012. Institui a Política Nacional de Saúde do Trabalhador e da Trabalhadora. Diário Oficial da União; Brasília, 24 Ago 2012.

6. Camelo SHH, Rocha FLR, Mininel VA, Santos APA, Garcia AB, Scozzafave MCS. Trabalhador de saúde: formas de adoecimento e estratégias de promoção à saúde. Revista Eletrônica Gestão \& Saúde. 2014;5(3):2220-29.

7. Glanzn $\mathrm{CH}$, Olschowsky A. A ambiência e sua influência no trabalho de equipes de saúde da família. Rev Bras Crescimento Desenvolv Hum. 2017;5(1):7-14.

8. Sangaletti J, Ceretta LB, Soratto MT. Ansiedade dos enfermeiros da estratégia saúde da família. RIES. 2018;7(1):234-48.

9. Leonelli LB, Andreoni S, Martins P, Kozasa EH, Salvo VL, Sopezki D, et al. Estresse percebido em profissionais da Estratégia Saúde da Família. Rev Bras Epidemiol. 2017;20(2):286-98.

10. Fonseca FF, Costa FM, Lima CA, Silva SSS, Alves JP, Carneiro JA. Caracterização do risco ocupacional entre trabalhadores da Estratégia Saúde da Família. Rev Bras Pesq Saúde. 2015;17(2):89-97.

11. Ministério da Saúde (BR). Política Nacional de Promoção da Saúde - PNaPS: revisão da Portaria MS/GM nº 687. Brasília: Ministério da Saúde; 2014.

12. Ministério da Saúde (BR). Secretaria de Gestão do Trabalho e da Educação na Saúde. Departamento de gestão da Educação na Saúde. Política de educação e desenvolvimento para o SUS: caminhos para a educação permanente e polos de educação permanente em saúde. Brasília: Ministério da Saúde; 2004.

13. Pinto HA. Política Nacional de Educação Permanente em Saúde: aprender para transformar. In: Gomes LB, Barbosa MG, Ferla AA, organizadores. A educação permanente em saúde e as redes colaborativas: conexões para a produção de saberes e práticas. Porto Alegre: Rede UNIDA; 2016. p. 23-65.

14. Ministério da Saúde (BR). Residência Multiprofissional em Saúde: experiências, avanços e desafios. Brasília: Ministério da Saúde; 2006.

15. Silva LB. Residência Multiprofissional em Saúde no Brasil: alguns aspectos da trajetória histórica. Rev Katálysis. 2018;21(1):200-9.

16. Brasil. Ministério da Saúde. Portaria $n^{\circ} 1.248$, de 24 de junho de 2013. Institui a Estratégia de qualificação das redes de atenção à saúde (RAS) por meio do incentivo à formação de especialistas na modalidade 
residência médica em áreas estratégicas do Sistema Único de Saúde (SUS). Diário Oficial da União; Brasília, 24 de Junho de 2013.

17. Fagundes NC, Rangel AGC, Carneiro TM, Castro LMC, Gomes BS. Educação permanente em saúde no contexto do trabalho da enfermeira. Rev Enferm UERJ. 2016;24(1):e11349.

18. Spink MJ, Menegon VM, Medrado B. Oficinas como estratégia de pesquisa: articulações teóricometodológicas e aplicações ético-políticas. Psicol Soc. 2014;26(1):32-43.

19. Pinheiro R, Gerhardt TE, Ruiz ENF, Silva AG Junior. O "estado do conhecimento" sobre os itinerários terapêuticos e suas implicações teóricas e metodológicas na Saúde Coletiva e integralidade do cuidado. Rio de Janeiro: CEPESC; 2016.

20. Heckert ALE, Neves CA. Modos de formar e modos de intervir: quando a formação se faz potência de produção de coletivo. In: Pinheiro R, Barros MEB, Motta R, organizadores. Trabalho em equipe. Rio de Janeiro: ABRASCO/IMS/UERJ; 2007. p. 145-60.

21. Ministério do Planejamento (BR). Unidade Básica de Saúde [Internet]. 2017. [acesso em 2019 Abr 17]. Disponível em: http://www.pac.gov.br/infraestrutura-social-e-urbana/ubs-unidade-basica-de-saude

22. Domingos CM, Nunes EFPA, Carvalho BG. Potencialidades da residência multiprofissional em saúde da família: o olhar do trabalhador de saúde. Interface [Internet]. 2015 [acesso em 2019 Abr 12];19(55):1221-32. Disponível em http://www.scielo.br/scielo.php?script=sci_arttext\&pid=S1414-32832015000401221

23. Rosa FM, Weiller TH, François APW, Beites LS, Silveira D, Righi LB. O olhar das equipes de referência sobre o trabalho realizado pelo apoio matricial. Rev Enf UFSM [Internet]. 2011 [acesso em 2019 Abr 17];1(3):37785. Disponível em: https://periodicos.ufsm.br/reufsm/article/view/3368/2405

24. Ceccim RB, Dallegrave D, Amorim ASL, Portes VM, Amaral BP. EnSiQlopédia das Residências em Saúde: Série vivências em saúde. Porto Alegre: Rede UNIDA; 2018.

25. Esposito T, Munoz-Castro FJ, Herrera-Usagre M, Perianez-Vega M. Fiabilidad y validez para un cuestionario de satisfaccion con la formacion continuada en salud: el cuestionario de satisfaccion del discente. FEM. 2015;18(3):197-203.

26. Silva LAA, Soder RM, Petry L, Oliveira IC. Educação permanente em saúde na atenção básica: percepção dos gestores municipais de saúde. Rev Gaúcha Enferm. 2017;38(1):e58779.

27. Brasil. Ministério da Saúde. Consulta pública $n^{\circ} 3$, de 17 de maio de 2011. Diretrizes da Política Nacional de Promoção da Saúde do Trabalhador do SUS. Artigo 34, inciso II, e artigo 59 do Decreto $n^{\circ} 4.176$, de 28 de março de 2002, minuta de portaria que aprova no âmbito do sistema Único de Saúde (SUS) [Internet]. 2011 [acesso em 2019 Abr 17]. Disponível em: http://bvsms.saude.gov.br/bvs/saudelegis/gm/2011/ cop0003_17_05_2011.html

\author{
Endereço do primeiro autor: \\ Luana Fagundes Galiski \\ Universidade do Vale do Itajaí - UNIVALI \\ Rua Uruguai, 458 \\ Bairro: Centro \\ CEP: 88302-901 - Itajaí - SC - Brasil \\ E-mail: luanafagundes580@hotmail.com
}

\author{
Endereço para correspondência: \\ Carlos Alberto Severo Garcia Júnior \\ Universidade Federal de Santa Catarina - UFSC \\ Rodovia Governador Jorge Lacerda, 3201/ Km35,4 \\ Bairro: Jardim das Avenidas \\ CEP: 88906-072 - Araranguá - SC - Brasil \\ E-mail: carlosgarciajunior@hotmail.com
}

Como citar: Galiski LF, Garcia CAS Júnior, Cunha LB, Bolognini T, Campos V. Oficinas de cuidado: um relato de experiência com os trabalhadores da saúde da família. Rev Bras Promoç Saúde. 2019;32:8842. 